\title{
Th17/Treg ratio derived using DNA methylation analysis discriminates allergen-induced early from dual asthmatic responses
}

\author{
Amrit Singh ${ }^{1 *}$, Masatsugu Yamamoto', Jian Ruan', Jung Young Choi', Gail M Gauvreau², Paul M O'Byrne², \\ Sven Olek ${ }^{3}$, Ulrich Hoffmueller ${ }^{3}$, Christopher Carlsten ${ }^{4}$, J Mark FitzGerald ${ }^{4}$, Louis-Philippe Boulet ${ }^{5}$, Scott J Tebbutt ${ }^{1}$ \\ From Canadian Society of Allergy and Clinical Immunology Annual Scientific Meeting 2013 \\ Toronto, Canada. 3-6 October 2013
}

\section{Background}

Atopic allergic asthmatic individuals experience acute bronchoconstriction (early response) upon allergen exposure. Several hours after the initial exposure, some individuals exhibit a chronic late phase (dual responders, DRs) whereas others do not (early responders, ERs). The purpose of this study is to determine changes in Th17 and regulatory $\mathrm{T}$ (Treg) cell numbers and their associated gene expression profiles in whole blood between allergen-induced ERs and DRs.

\section{Methods}

14 participants with mild, atopic asthma (8 ERs and 6 DRs) underwent a cat allergen inhalation challenge as part of the AllerGen Clinical Investigator Collaborative. Whole blood was collected immediately prior to challenge (pre) and 2 hours post-challenge. DNA methylation analysis was used to measure the frequency of Th17, Treg, B and T cells (Epiontis, Germany). Whole blood transcriptome profiling was performed using Affymetrix GeneChip ${ }^{\circledR}$ Human Gene 1.0 ST Arrays. Statistical analysis was performed using $\mathrm{R}$.

\section{Results}

Sum of the T cell and B cell frequencies obtained using the methylation assays strongly correlated $(r=0.95)$ with the lymphocyte frequency obtained using a hematolyzer. Allergen inhalation did not significantly $(p>0.05)$ change Th17, Treg, B and T cell counts between ERs and DRs. However, the Th17/Treg ratio was significantly $(\mathrm{p}=0.03)$ different between ERs and DRs post challenge. 199 genes positively correlated with Th17 cells at an FDR of 5\%. 463 genes positively correlated with Treg cells at an FDR of 5\%. Th17 genes were inversely correlated with Treg genes.

\section{Conclusions}

Th17/Treg ratio derived using DNA methylation analysis discriminates allergen-induced early from dual asthmatic responses. The inverse correlation between Th17 genes and Treg genes may be indicative of the inflammatory or suppressive phenotypes of these cells.

\section{Authors' details \\ 'James Hogg Research Centre, St. Paul's Hospital, University of British Columbia, Vancouver, British Columbia, V6Z 1Y6, Canada. ${ }^{2}$ Department of Medicine, McMaster University, Hamilton, Ontario, L8S 4L8, Canada. ${ }^{3}$ Epiontis $\mathrm{GmbH}$, Berlin, Germany. ${ }^{4}$ Vancouver Coastal Health Research Institute, Vancouver General Hospital, Vancouver, British Columbia, V5Z 1M9, Canada. ${ }^{5}$ Centre de Pneumologie de L'Hopital, Université Laval, Sainte-Foy, Quebec, G1V 0B4, Canada.}

Published: 3 March 2014
doi:10.1186/1710-1492-10-S1-A46
Cite this article as: Singh et al: Th17/Treg ratio derived using DNA methylation analysis discriminates allergen-induced early from dual asthmatic responses. Allergy, Asthma \& Clinical Immunology 2014 10(Suppl 1):A46.

\footnotetext{
* Correspondence: amrit.singh@hli.ubc.ca

'James Hogg Research Centre, St. Paul's Hospital, University of British

Columbia, Vancouver, British Columbia, V6Z 1Y6, Canada

Full list of author information is available at the end of the article
} 